\title{
Correction: Minimal Intensity Physical Activity (Standing and Walking) of Longer Duration Improves Insulin Action and Plasma Lipids More than Shorter Periods of Moderate to Vigorous Exercise (Cycling) in Sedentary Subjects When Energy Expenditure Is Comparable
}

\section{The PLOS ONE Staff}

There is an error in the third sentence of the Methodology/ Principle Findings section of the Abstract. The correct sentence is: The sitting and exercise regime had comparable numbers of sitting hours; compared to the exercise regime, the minimal intensity PA regime had a higher estimated daily energy expenditure (238kcal/ day).

There is an error in the sixth sentence of the Methodology/ Principle Findings section of the Abstract. The correct sentence is: In the sitting regime, daily energy expenditure was about 300 and 500 kcal lower than in the exercise and minimal intensity PA regimes respectively.

There is an error in the second sentence of the Physical activity, postural allocation and energy intake and expenditure segment of the Results section. The correct sentence is: During the exercise regime all participants had a daily, 1 hour bicycle exercise with a mean increase of heart rate of $52 \pm 3$ beats/min, resulting in an estimated energy expenditure of $367 \pm 5 \mathrm{kcal}$.

There is an error in the penultimate sentence of the Physical activity, postural allocation and energy intake and expenditure segment of the Results section. The correct sentence is: Compared to the sitting regime, estimated DEE was about 300 and $500 \mathrm{kcal}$ higher during the exercise and minimal intensity PA regimes respectively; estimated DEE was $238 \mathrm{kcal} /$ day higher during the minimal intensity PA in comparison to the exercise regime: 2248 vs $2486 \mathrm{kcal} /$ day $(\mathrm{p}<0.001)$.

There is an error in the fourth sentence of the second paragraph in the Discussion section. The correct sentence is: Participants followed the imposed regimes well, and as sleeping time was the same in the three regimes, estimated DEE was increased by 314 kcal during the exercise regime and with 553 kcal during the minimal intensity PA regime.

There is an error in Table 2. In the Daily Energy Expenditure row, the data is incorrect in the Exercise regime column and the $\mathrm{p}$ exerc vs MIPA column. Please see the corrected table below.
Citation: The PLOS ONE Staff (2014) Correction: Minimal Intensity Physical Activity (Standing and Walking) of Longer Duration Improves Insulin Action and Plasma Lipids More than Shorter Periods of Moderate to Vigorous Exercise (Cycling) in Sedentary Subjects When Energy Expenditure Is Comparable. PLoS ONE 9(8): e105135. doi:10.1371/journal.pone.0105135

Published August 5, 2014

Copyright: () 2014 The PLOS ONE Staff. This is an open-access article distributed under the terms of the Creative Commons Attribution License, which permits unrestricted use, distribution, and reproduction in any medium, provided the original author and source are credited. 
Table 2. Daily energy intake and expenditure, time spent in activity categories and glucose metabolism and plasma lipids.

\begin{tabular}{|c|c|c|c|c|c|c|c|}
\hline & Sitting regime & Exercise regime & $\begin{array}{l}\text { Minimal intensity } \\
\text { PA regime }\end{array}$ & p-value & $\begin{array}{l}\text { p sit vs } \\
\text { exerc. }\end{array}$ & $\begin{array}{l}\text { p sit vs } \\
\text { MIPA }\end{array}$ & $\begin{array}{l}\text { p exerc vs } \\
\text { MIPA }\end{array}$ \\
\hline $\begin{array}{l}\text { Daily Energy intake } \\
\text { (kcal, } n=18)\end{array}$ & $1539(427)$ & $1477(352)$ & 1394(292) & 0.136 & & & \\
\hline Proteins $(g, n=18)$ & $61.1(14.8)$ & $59.7(13.5)$ & $55.6(13.4)$ & 0.165 & & & \\
\hline Fat $(g, n=18)$ & $54.5(14.7)$ & $50.2(19.6)$ & $50.1(12.2)$ & 0.248 & & & \\
\hline Carbohydrates $(g, n=18)$ & 199.0(68.9) & 196.7(48.9) & $180.0(51.2)$ & 0.227 & & & \\
\hline $\begin{array}{l}\text { Daily Energy Expenditure } \\
\text { (kcal, } n=16)\end{array}$ & 1934(88) & 2248(93) & $2486(121)$ & $<0.001$ & $<0.001$ & $<0.001$ & $<0.001$ \\
\hline Sitting time $(h r, n=17)$ & $13.6(1.2)$ & $12.7(1.7)$ & $7.4(1.3)$ & $<0.001$ & 0.002 & $<0.001$ & $<0.001$ \\
\hline Standing time $(h r, n=17)$ & $0.99(0.50)$ & $1.08(0.48)$ & $3.08(0.88)$ & $<0.001$ & 0.166 & $<0.001$ & $<0.001$ \\
\hline $\begin{array}{l}\text { Active-not exercise time } \\
(\mathrm{hr}, \mathrm{n}=17)\end{array}$ & $0.81(0.29)$ & $1.01(0.26)$ & $4.85(0.63)$ & $<0.001$ & 0.001 & $<0.001$ & $<0.001$ \\
\hline Sleeping time $(h r, n=17)$ & $8.58(0.74)$ & $8.17(1.37)$ & $8.65(0.93)$ & 0.200 & & & \\
\hline Steps/day $(n=16)$ & 4324(1485) & $6049(1402)$ & $27590(3724)$ & $<0.001$ & $<0.001$ & $<0.001$ & $<0.001$ \\
\hline $\begin{array}{l}\text { Triglycerides } \\
(\mathrm{mmol} / \mathrm{l} ; \mathrm{n}=18)\end{array}$ & $0.90(0.26)$ & $0.85(0.35)$ & $0.70(0.23)$ & 0.007 & 0.326 & 0.002 & 0.029 \\
\hline $\begin{array}{l}\text { Total Cholesterol } \\
(\mathrm{mmol} / \mathrm{l} ; \mathrm{n}=18)\end{array}$ & $4.20(0.67)$ & $4.11(0.60)$ & $3.96(0.50)$ & 0.171 & & & \\
\hline $\begin{array}{l}\text { HDL-Cholesterol } \\
(\mathbf{m m o l} / \mathbf{l} ; \mathbf{n}=18)\end{array}$ & $1.26(0.34)$ & $1.27(0.28)$ & $1.30(0.30)$ & 0.686 & & & \\
\hline $\begin{array}{l}\text { Non-HDL-Cholesterol } \\
(\mathrm{mmol} / \mathrm{l} ; \mathrm{n}=18)\end{array}$ & $2.94(0.47)$ & $2.84(0.57)$ & $2.65(0.48)$ & 0.011 & 0.275 & 0.007 & 0.048 \\
\hline $\begin{array}{l}\text { LDL-Cholesterol } \\
\text { (mmol//; } \mathrm{n}=18)\end{array}$ & $2.53(0.51)$ & $2.45(0.57)$ & $2.34(0.49)$ & 0.094 & & & \\
\hline Apo A-I (g/l; $n=18)$ & $1.57(0.24)$ & $1.57(0.21)$ & $1.55(0.21)$ & 0.905 & & & \\
\hline Apo B $(g / l ; n=18)$ & $0.75(0.12)$ & $0.70(0.16)$ & $0.69(0.14)$ & 0.022 & 0.052 & 0.005 & 0.627 \\
\hline $\begin{array}{l}\text { Insulin Sensitivity Index } \\
(n=17)\end{array}$ & $20.4(8.2)$ & 22.8(9.9) & $26.3(11.7)$ & 0.052 & 0.246 & 0.051 & 0.036 \\
\hline $\begin{array}{l}\text { Fasting Glucose } \\
(\mathbf{m m o l} / \mathbf{m l} ; n=17)\end{array}$ & $4.6(0.4)$ & $4.5(0.3)$ & $4.5(0.4)$ & 0.681 & & & \\
\hline $\begin{array}{l}\text { Fasting Insulin } \\
\text { (mU/ml; } n=18)\end{array}$ & $11.5(9.0)$ & $9.4(4.4)$ & $8.5(4.0)$ & 0.310 & & & \\
\hline $\begin{array}{l}\text { AUC glucose } \\
(\mathrm{mmol} \mathrm{min} / \mathrm{ml} ; n=17)\end{array}$ & 715.7(135.7) & $765.8(115.9)$ & $754.9(141.8)$ & 0.171 & & & \\
\hline $\begin{array}{l}\text { AUC insulin } \\
(\mathrm{mU} \mathrm{min} / \mathrm{ml} ; n=18)\end{array}$ & $7752.0(3015.4)$ & $8320.4(5383.7)$ & 6727.3(4329.4) & 0.005 & 0.841 & 0.010 & 0.002 \\
\hline $\begin{array}{l}\text { AUC C-peptide } \\
(\mathrm{nmol} \mathrm{min} / \mathrm{l} ; n=18)\end{array}$ & 217.4(76.6) & 219.2(67.4) & 193.0(63.7) & 0.104 & & & \\
\hline
\end{tabular}

Plasma lipids, glucose, insulin and C-peptide levels were assessed in fasting state. Second, third and fourth column contain average values and standard deviations for each of the regimes. The fifth column represents the level of significance for repeated measurements ANOVA. Column six to eight give the statistical significance for pairwise comparisons of the regimes (Least Significant Differences, $\mathrm{p}$-values were not corrected for multiple testing). For pairwise comparing, $\mathrm{p}$-values less than 0.017 were considered significant.

doi:10.1371/journal.pone.0055542.t002

\section{Reference}

1. Duvivier BMFM, Schaper NC, Bremers MA, van Crombrugge G, Menheere PPCA, et al. (2013) Minimal Intensity Physical Activity (Standing and Walking) of Longer Duration Improves Insulin Action and Plasma Lipids More than Shorter Periods of Moderate to Vigorous Exercise (Cycling) in Sedentary Subjects When Energy Expenditure Is Comparable. PLoS ONE 8(2): e55542. doi: $10.1371 /$ journal.pone.0055542 